\title{
THE INFLUENCE OF LOCALIZED STRESSES AND STRAINS ON THE IODINE SCC BEHAVIOR OF ZIRCALOY-2 TUBING
}

\author{
B.N. NOBREGA, J.S. KING and G.S. WAS \\ The University of Michigan, Department of Nuclear Engineering, Ann Arbor, MI 48109, USA
}

\author{
R.B. ADAMSON \\ General Electric Company, Core Materials Testing, Vallecitos Nuclear Center, Pleasanton, CA 94566, USA
}

Received 24 July 1984 ; accepted 6 December 1984

\begin{abstract}
The segmented expanding mandrel test is modified to provide additional information on the localized stresses and strains during ramp-hold tests. A chamfered specimen is designed with four flats on the outer diameter for the purpose of creating sectors of variable wall thickness which establishes a more nearly plane strain state in the thinnest-gauge sections. Ramp-hold tests conducted in $40 \mathrm{MPa}$ of iodine at $325^{\circ} \mathrm{C}$ show that at a measured diametral strain of $0.8 \%$, chamfered specimens fail while regular tubing specimens require diametral strains of $2 \%$ or greater for failure. Calculations made with a two-dimensional, finite clements code show that at a measured diametral strain of $0.8 \%$, the local strains in the clad ID over the segment interfaces are nearly identical for the regular and chamfered samples while the local stress in the chamfered sample is $15 \%$ greater. Results suggest that the smaller diametral failure strain of the chamfered specimen at low measured diametral strains is mainly due to its near plane strain condition and the higher hoop stresses resulting therefrom.
\end{abstract}

\section{Introduction}

Pellet-cladding interaction (PCI) failures in Zircaloy-clad $\mathrm{UO}_{2}$ fuel rods are generally attributed to environmentally assisted cracking phenomena that combine the chemical aggressiveness of certain fission products (e.g. I, Cd) with the high local hoop stresses in cladding regions near radial fuel cracks or pellet-pellet interfaces during power ramps [1-3]. Such concentration (magnification) of stresses plays a key role in the $\mathrm{PCI}$ failure mechanism, for without it the mechanical conditions in power-ramped cladding would be insufficient to cause PCI failure [4]. In an effort to gain an understanding of the $\mathrm{PCI}$ phenomenon, much research has been concentrated on two types of laboratory tests commonly used to assess the stress corrosion cracking (SCC) susceptibility of Zircaloy tubing: the internally pressurized tubing test (IPTT) and the segmented expanding mandrel test (SEMT).

The internally pressurized tube test uses closed-end pressurization of a $15-20 \mathrm{~cm}$ long tubing sample with a reduced outer diameter in the gauge section to achieve a biaxial state of stress [5-9]; see fig. 1. An iodine-containing ampoule, placed between two volume-displacing rods inside the specimen breaks and releases iodine vapor as the tubing pressure increases. The stress state remains practically constant throughout the test and the diametral strain obtained at failure consistently falls in the $<0.5 \%$ range which is also typical of in-reactor defected rods $[5,6,10]$. However, the IPTT provides no stress-strain concentration in localized regions of the specimens, an important characteristic of pellet-cladding interactions. Furthermore, the internal pressurization makes this test a force-controlled technique, that is the specimen is loaded by an imposed pressure and not by displacement of internal components as in the actual fuel application.

Another widely used test is the segmented expanding

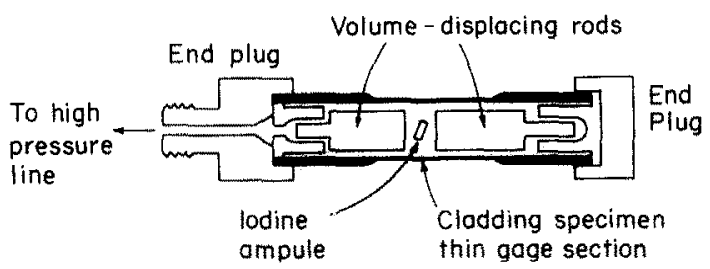

Fig. 1. Schematic of the internally pressurized tubing test. 


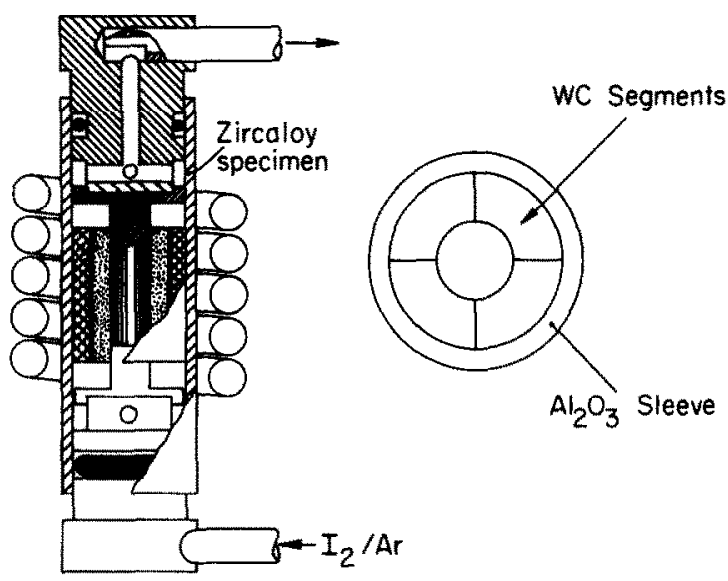

Fig. 2. Schematic of the segmented expanding mandrel test, (Ref. [11]).

mandrel technique (SEMT) [11-13]. In one version of this test [11], a reactor-grade zirconium slug is mechanically compressed by metallic plungers at a constant rate in a mechanical testing machine; see fig. 2. Four surrounding tungsten carbide segments are radially displaced against an $\mathrm{Al}_{2} \mathrm{O}_{3}$ sleeve that simulates the $\mathrm{UO}_{2}$ pellets. The sleeve breaks preferentially at the tungsten carbide interfaces, similar to the actual fuel situation. The SEMT thus affords some degree of stress-strain concentration in localized regions of the tube. It also has the advantage of being a displacement-controlled method. However, the local stresses and strains are not easily determined either experimentally or analytically. Moreover, the low plastic failure strains typical of PCIdefected rods are not generally reproduced by SEMT simulations, the failure strains being of the order of $2 \%$ or greater.

A comparison between the local mechanical effects in SEMT samples and actual fuel cladding reveals strong similarities in most aspects with one exception: while the latter is believed to be subjected to a near equibiaxial stress state $\left(\sigma_{\text {hoop }} \approx \sigma_{\text {axial }}\right)$ during a power ramp [4], the laboratory specimens are believed to be closer to a uniaxial stress condition $\left(\sigma_{\text {axial }}=0\right)$. The objective of this study is to identify the nature of the discrepancy in failure strain between the SEMT results and PCI-defected fuel rods by working with the hypothesis that a plane-strain state coupled with high local stresses can induce low diametral strain failures in SEMT specimens typical of PCI-defected fuel rods. Although all experiments were performed in an iodine-containing atmosphere, the role of iodine in PCI failure is not called into question. In all of the tests herein reported, the iodine atmosphere was constant.

\section{Experiment}

\subsection{Design}

Several aspects of the conventional segmented expanding mandrel test [11] are modified in order to provide greater information on the local cladding stresses and strains. The modifications are described in detail in Ref. [14] and are summarized in table 1. The design used in this study is shown in fig. 3. The internal components consists of a crystal bar zirconium slug surrounded by four $\mathrm{Al}_{2} \mathrm{O}_{3}$ segments with an intersegment gap of $445 \mu \mathrm{m}$.

Three specimen geometries are used - a regular tube, a chamfered tube and a thin-walled tube. The chamfered design has four flats on the OD which create sectors of variable wall thickness for the purpose of establishing a more nearly plane strain state in the thinnest-gauge sections. The $\mathrm{Al}_{2} \mathrm{O}_{3}$ segments are aligned so that the gaps between segments are centered on the midpoint of the flats in the tubing. The thin-wall samples have the same thickness as the minimum section of the chamfered sample. Both the regular and thin-wall specimens serve as control samples to separate out of the effect of wall thickness.

\subsection{Materials}

The specimens were cut from $60 \%$ cold-worked, unirradiated Zircaloy-2 tubes of the $8 \times 8$ BWR design manufactured and supplied by the General Electric Company. Mechanical properties of these tubes at $325^{\circ} \mathrm{C}$ are given in table 2. Standard back-reflection X-ray diffraction analyses of the midwall and outer surface of

Table 1

Summary of modifications made to the segmented expanding mandrel technique

\begin{tabular}{|c|c|c|}
\hline Component & $\begin{array}{l}\text { Conventional } \\
\text { design (ref. [11]) }\end{array}$ & $\begin{array}{l}\text { Modified } \\
\text { design }\end{array}$ \\
\hline Central slug & $\mathrm{Zr}$ & $\mathrm{Zr}$ \\
\hline Segments & WC & $\mathrm{Al}_{2} \mathrm{O}_{3}$ \\
\hline Sleeve & $\mathrm{Al}_{2} \mathrm{O}_{3}$ & none \\
\hline Sample & regular & $\begin{array}{l}\text { regular, chamfered, } \\
\text { thin-wall }\end{array}$ \\
\hline Heating & RF & resistance furnace \\
\hline
\end{tabular}




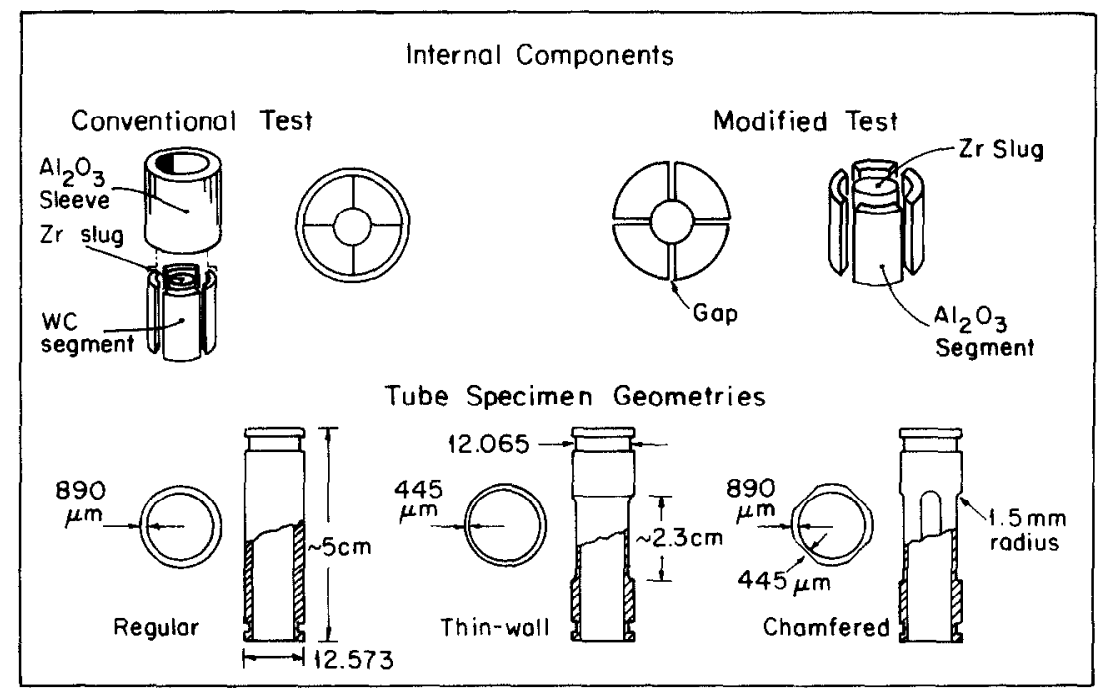

Fig. 3. Segmented expanding mandrel test design used in this study.

the cold-worked unirradiated (CWUE) cladding yielded the $f$-parameters and pole figures given in table 2 and fig. 4 , respectively. The usual basal pole texture of split peaks about $30^{\circ}$ from the radial direction is apparent and no appreciable difference is noticeable between the analyzed surfaces.

The internal wall surface of the tubes was examined by scanning electron microscopy (SEM). A large surface density of small fissures preferentially oriented in the

Table 2

Material characteristics of Zircaloy specimens

Dimensions: $O D=12.573 \mathrm{~mm}$

ID $=10.793 \mathrm{~mm}$

Metallurgical state: $60 \%$ cold worked

Mechanical properties at $325^{\circ} \mathrm{C}$ :

Young's modulus $=79.165 \mathrm{GPa}$

Poissons's ratio $\quad=0.43$

Yield strength $\quad=353 \mathrm{MPa}$

Ultimate strength $\quad=513 \mathrm{MPa}$

(0002) Plane orientation parameters:

Middle surface

\begin{tabular}{lll}
\hline$f_{r}$ & $f_{z}$ & $f_{\theta}$ \\
\hline 0.618 & 0.052 & 0.328 \\
0.610 & 0.051 & 0.339 \\
\hline
\end{tabular}

Rockwell-B number (ID surface): 99 circumferential direction was observed. This so-called open grain structure results from the cladding manufacturing procedures and is typical of unetched or lightly elched Zircaloy lubes.

\subsection{Procedure}

The samples are heated with a miniature resistance furnace through which a horizontal slot is cut for diametral extensometry; see fig. 5. For all samples, diametral strain is measured by fixing the extensometer contact points across the diameter connecting segment interfaces. The test fixture is brought up to temperature $\left(325^{\circ} \mathrm{C}\right)$ and is allowed approximately $30 \mathrm{~min}$ for equilibration. A mixture of iodine and argon is flowed through the specimen providing an iodine vapor pressure of $40 \mathrm{MPa}$ at room temperature. The ramp-hold (RH) test is started by moving the actuator in compression at a constant rate of $12.7 \mu \mathrm{m} / \mathrm{min}$. The resulting load applied to the zirconium slug and the deflection of the extensometer are plotted on an $X-Y$ recorder. The actuator is stopped and held fixed when a preset diametral deformation (ramp strain) is reached. Tests are interrupted either when specimen failure is detected by a sharp decrease in the applied load or after a predetermined time limit ( $\geqslant 10 \mathrm{~h})$ is reached. Post-test examination includes diametral strain measurement at the segment midpoints and interfaces, and fractographic analysis. 


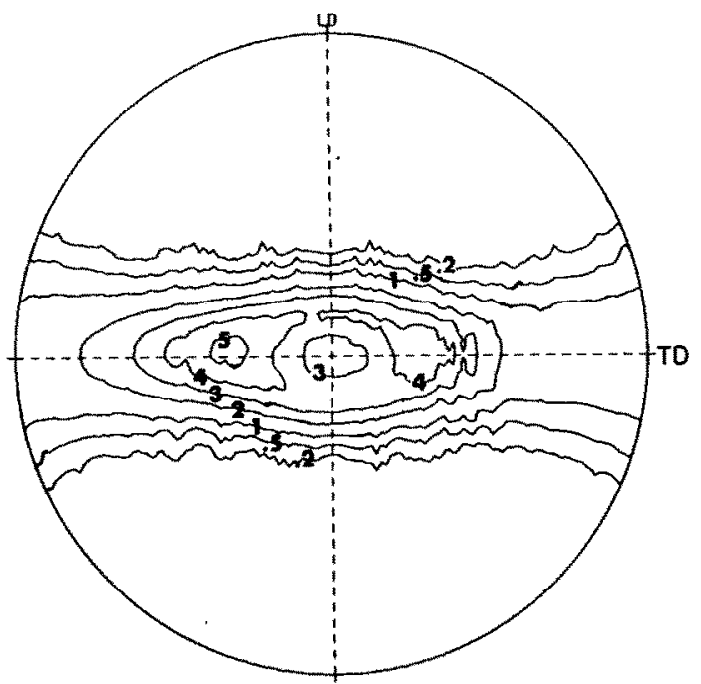

MIDWALL SURFACE

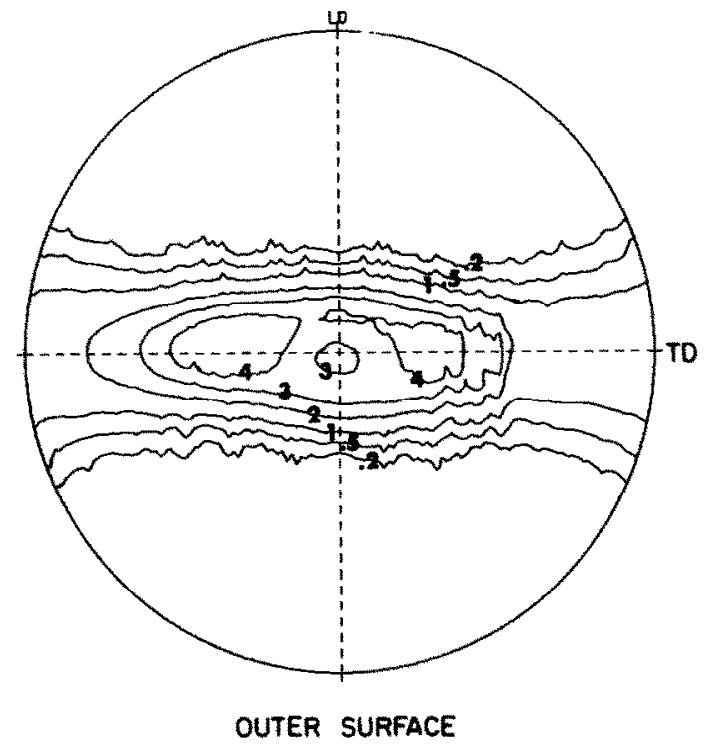

Fig. 4. Radial direction pole figures of the (0002) plane of CWUE tubing.

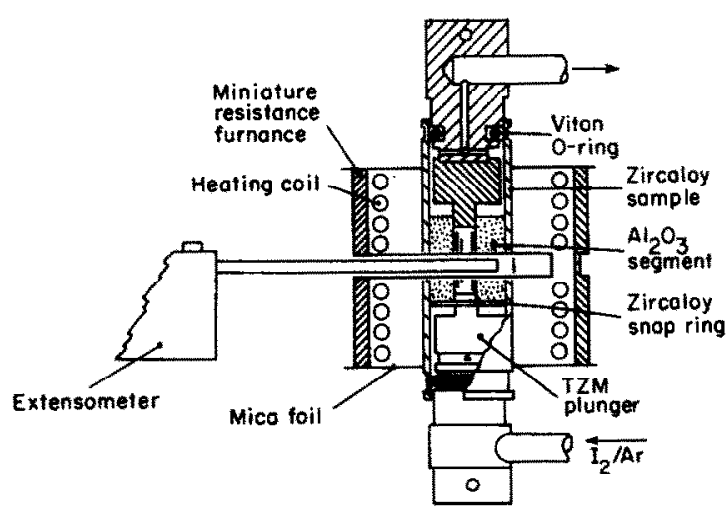

Fig. 5. Details of the complete modified segmented expanding mandrel test apparatus.

\section{Results}

The results of ramp-hold tests conducted on regular, thin-wall, and chamfered tubing samples are given in table 3. Diametral strains measured from post-test examination are also given in table 3. Regular specimens were ramped to strain levels of $0.8 \%, 1.0 \%$ and $2.0 \%$. In no case was a failure incurred for a diametral strain less than $2.0 \%$. This diametral failure strain is typical and has been commonly observed whether the test is conducted on the present modified SEMT device or on the conventional SEMT design [15], and it is also typical of conventional SEMT tests of irradiated Zircaloy-2 tubing [16].

By contrast seven of the chamfered samples failed after diametral ramp strains of $0.82 \%$ to $0.91 \%$. Three chamfered samples with strains between $0.45 \%$ and $0.63 \%$ did not fail, suggesting a failure threshold between $0.63 \%$ and $0.8 \%$. This corresponds to a plastic strain beyond elastic deformation of between $0.25 \%$ and $0.4 \%$, which is consistent with typical plastic diametral strains in PCI-defected fuel rods.

All seven of the thin-wall samples listed in table 3 had post-test diametral strains larger than $0.71 \%$. However, only one sample failed and for this the total diametral strain was $1.16 \%$, the largest for all thin-wall samples.

In all cases, specimen failures occurred at a circumferential position directly over the gaps between adjacent $\mathrm{Al}_{2} \mathrm{O}_{3}$ segments. Typically they were narrow axial splits 3 to $10 \mathrm{~mm}$ long in the central gauge portion loaded by the internal components. Fig. 6 shows the typical characteristics of a failed regular specimen ramped to $2.0 \%$ diametral strain. The fracture is predominantly brittle with the usual 'torn' appearance of heavily cold-worked Zircaloys cracked in iodine atmospheres. A small fraction of the failure surface near the outer diameter shows coalesced microvoids characteristic of ductile fractures and typical of the final propagation stages of stress corrosion cracks. 
Table 3

Results of ramp-hold tests at $325^{\circ} \mathrm{C}$ on regular, chamfered and thin-wall specimens

\begin{tabular}{lllllc}
\hline $\begin{array}{l}\text { Specimen } \\
\text { type }\end{array}$ & $\begin{array}{l}\text { Number of } \\
\text { tests }\end{array}$ & $\begin{array}{l}\text { Applicd } \\
\text { diametral } \\
\text { strain(\%) }\end{array}$ & Failure? & $\begin{array}{l}\text { Total diametral } \\
\text { failure strain(\%) }\end{array}$ & $\begin{array}{l}\text { Failure } \\
\text { time(min) }\end{array}$ \\
\hline Regular & 1 & 2.0 & yes & 2.15 & 120 \\
Regular & 1 & 1.0 & no & - & - \\
Regular & 1 & 0.8 & no & - & $120(\mathrm{avg})$ \\
Chamfered & 7 & 0.8 & yes & - & - \\
Chamfered & 3 & $0.8 *$ & no & - & - \\
Thin-wall & 6 & 0.8 & yo & 0.87 & 113 \\
Thin-wall & 1 & 0.8 & yes & & \\
\hline
\end{tabular}

* Actual ramp strains were inadvertantly limited to $0.45 \%, 0.61 \%$ and $0.63 \%$ for these three specimens.

Chamfered specimens ramped to measured diametral strains of $0.8 \%$ exhibited similar fracture features with slightly reduced ductile fractions. This suggests that despite their reduced wall thickness, the thin-gauge re- gions were not mechanically overloaded by the ramp. Nevertheless, some local necking was usually seen at the crack location; see fig. 7 .

It is noteworthy that cracks usually initiated at the

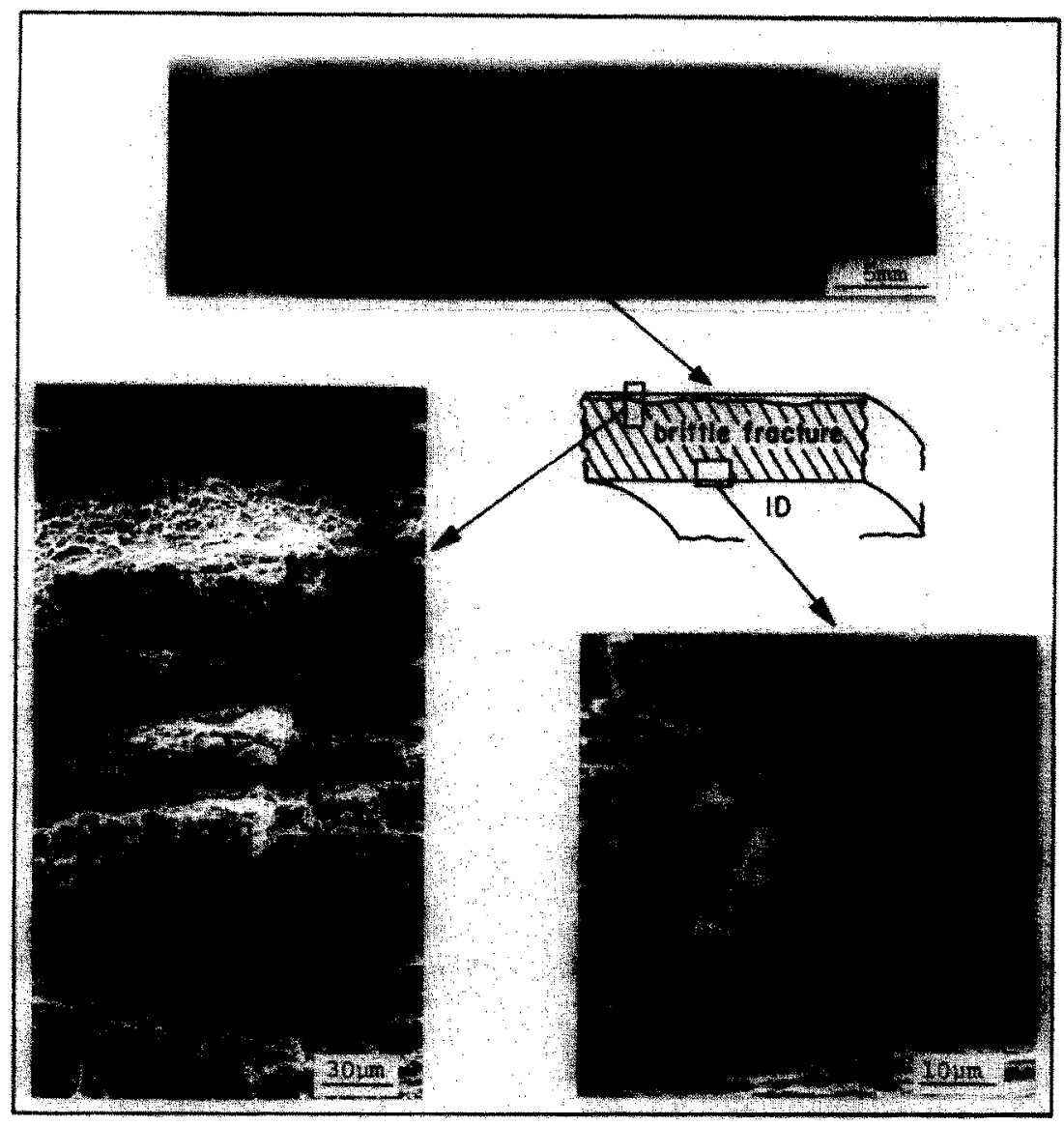

Fig. 6. Features of a fractured CWUE regular specimen. 


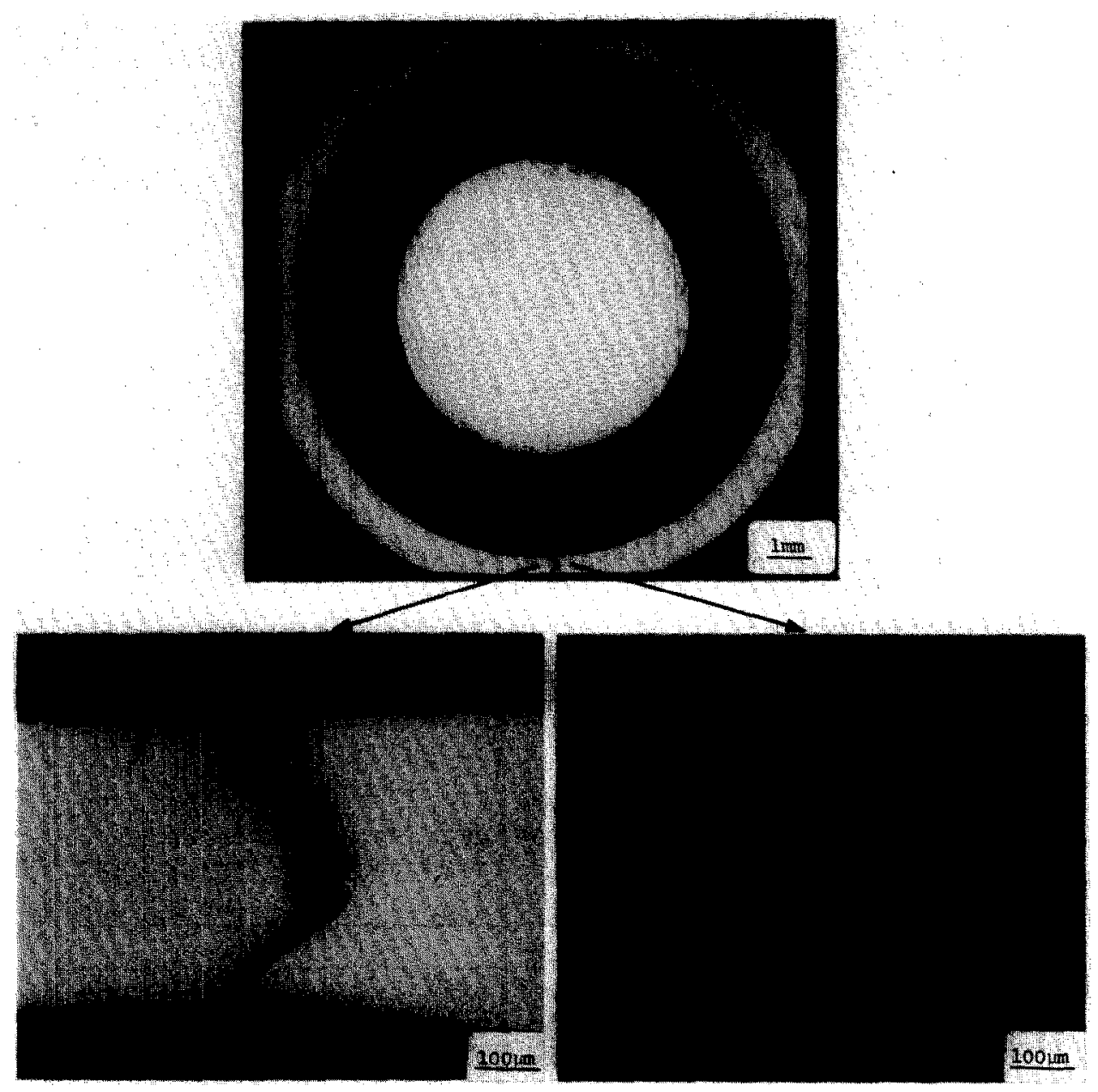

Fig. 7. Transverse section of a cracked CWUE chamfered specimen.

edge of one of the $\mathrm{Al}_{2} \mathrm{O}_{3}$ segments and followed an approximately radial direction a short distance (100 $\mu \mathrm{m})$ into the cladding wall and then proceeded on a zigzag path at nearly $60^{\circ}$ to the tube surface normal. This fracture morphology probably results from the combined effects of texture and cracking rates. At the initiation stage, cracking is controlled by the bulk hoop stress in the specimen wall $[17,18]$ and therefore follows a radial path at relatively low speeds. As the crack grows, the increasing stress intensity $\left(K_{\mathrm{I}}\right)$ at its tip takes control of the crack propagation process [17] resulting in high cracking rates. Accordingly, transgranular separation (cleavage) along quasi-basal planes of the hexagonal structure of Zircaloys becomes increasingly favored, and because of the $30^{\circ}$ split peak texture of the material the observed segmented fracture path ensues. Such in- terpretations imply that no significant hoop stress relaxation occurred in the tubing during the short hold periods $(<1 \mathrm{~h})$ required to fracture chamfered CWUE samples.

Comparison of these results for the three sample types lead to some simple observations. First, the comparison of thin-wall and chamfered-wall failure behavior shows that the lower diametral failure strains of the latter are not simply due to the reduced tubing wall thickness, per se, but are in some way brought about by the variable wall thickness geometry. Second, the onset of failures in the chamfered samples at measured diametral strains much lower than for conventional SEMT tests must also be attributed to an altered local stress-strain state over the $\mathrm{Al}_{2} \mathrm{O}_{3}$ segment gaps. Specifically, the lower-strain failures are postulated to be due 
to the creation of a condition near circumferential plane strain as opposed to a condition near plane stress as in the conventional SEMT tests. Such a plane strain condition is imposed in actual reactor service.

To verify the contention that there is a difference in the stress-strain state between the different sample geometries, chamfered and thin-wall specimens were instrumented with strain-guage rosettes and loaded to $0.8 \%$ diametral strain at room temperature. In a pure plane stress state, $\varepsilon_{z} / \varepsilon_{\theta}=-\nu=-0.43$ and in pure plane strain, $\varepsilon_{z} / \varepsilon_{\theta}=0$. Measurements revealed that for the thin-wall tubing $\varepsilon_{z} / \varepsilon_{\theta}=-0.46$ and for the chamfered design, $\varepsilon_{z} / \varepsilon_{\theta}=-0.21$. Thus, the uniform-wall designs are indeed in a state of plane stress while the chamfered design is substantially closer to plane strain.

Although our own strain-guage measurements confirm the nature of the strain state, the variation in local strains cannot be directly observed. They can, however, be calculated using finite-elements methodology. Therefore, the three types of SEMT specimens were analyzed by a two-dimensional finite-elements code, SAF2D $[19,20]$, for the purpose of determining the local stresses and strains at the segment interfaces corresponding to measured values of diametral strain. In what follows, all stresses and local strains are calculated, not measured.

\section{Analysis}

The SAF2D finite-elements code models displacement-controlled loading of the SEMT samples, the interfacial friction between the $\mathrm{Al}_{2} \mathrm{O}_{3}$ segments and the cladding, and cladding creep. The code accepts either axisymmetric $(r-z)$ or transverse $(r-\theta)$ finite element models. The latter requires either a plane stress $\left(\sigma_{z}=0\right)$, a plane strain $\left(\varepsilon_{z}=0\right)$ or a generalized plane strain ( $\varepsilon_{z}=$ constant $)$ condition. SAF2D models can comprise triangular, quadrilateral or special uniaxial elements. The latter can be used to simulate pellet-pellet and pellet-cladding contact including the effects of friction. Both the thermal-mechanical fuel and cladding responses are calculated.

The three sample geometries were modeled as shown in fig. 8. Owing to the circumferential symmetry of the problem, only $45^{\circ}$ sectors were modeled. The concentration of small $(25 \mu \mathrm{m} \times 56 \mu \mathrm{m})$ quadrilateral elements in the cladding over the slit between adjacent $\mathrm{Al}_{2} \mathrm{O}_{3}$ segments minimized numerical errors and improved the accuracy of the calculated local stresses and strains.

Ramp-hold SEMT tests were simulated by imposing 58 radial displacement increments ( $2 \mu \mathrm{m}$ each) on node A, fig. 8, so that the displacement history of node B would match the mcasured laboratory test results. As described earlier, the extensometer measurements are made at node $B$.
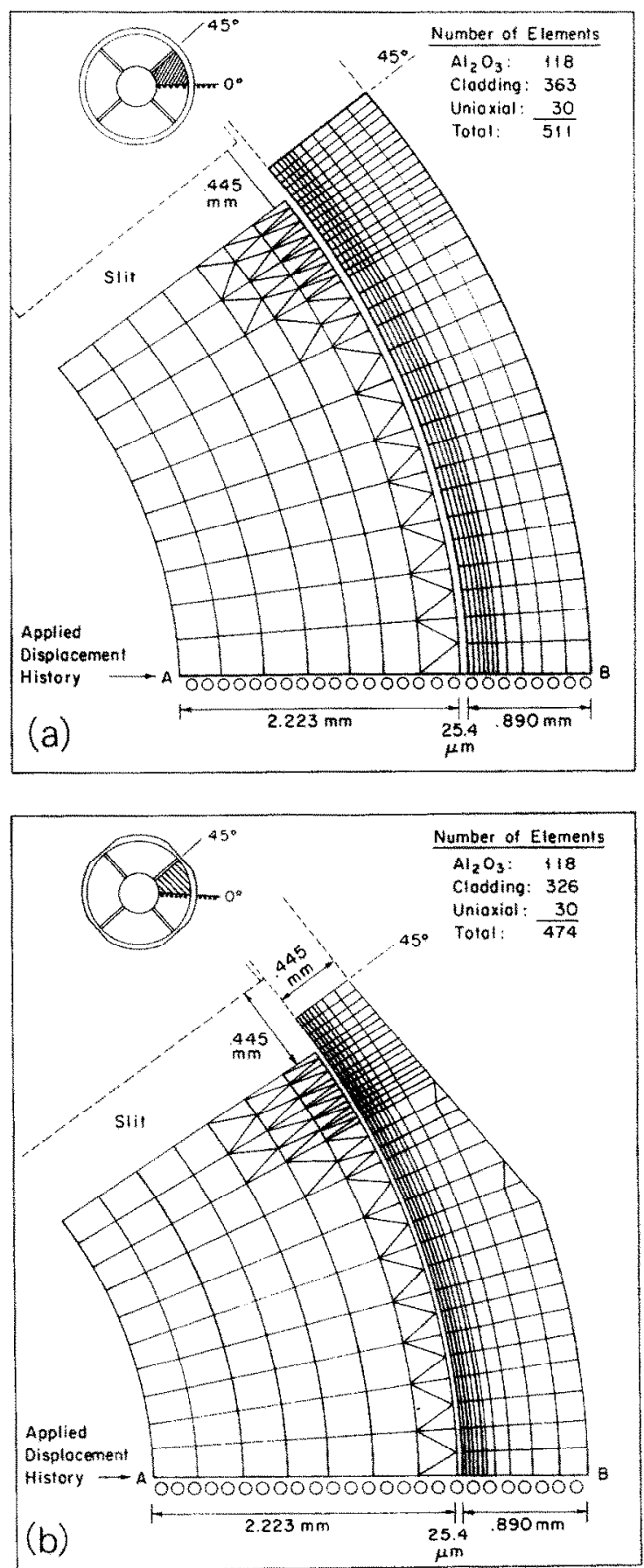


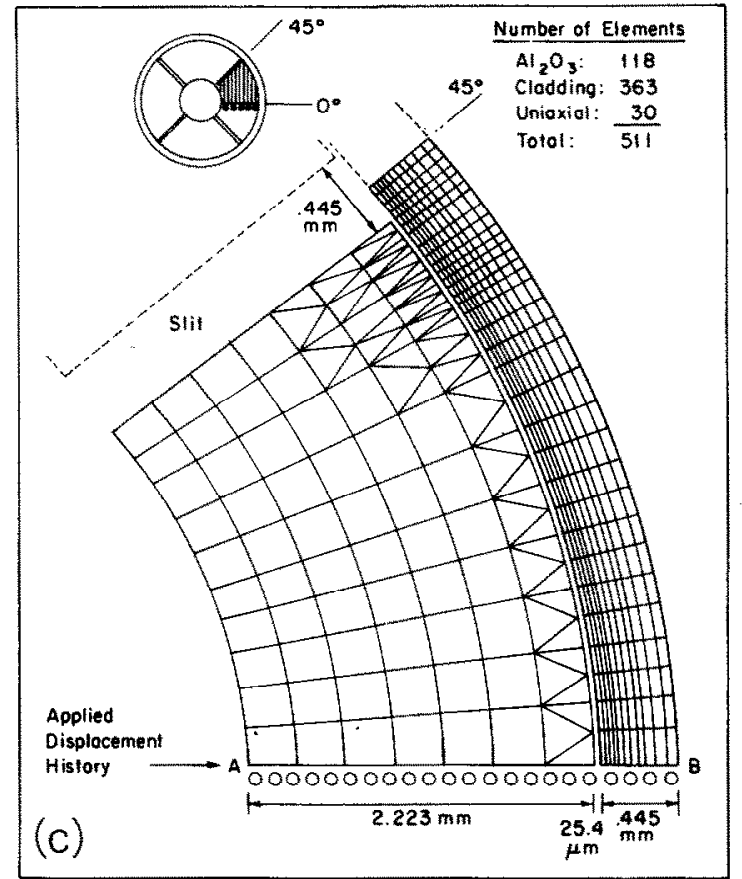

Fig. 8. The transverse finite element model of the (a) regular, (b) chamfered and (c) thin-wall specimen designs.

Sliding boundary conditions were applied to the nodes on the $\mathrm{AB}$ symmetry line and to the cladding nodes on the $45^{\circ}$ edge, that is these nodes were allowed to move radially only. The calculations were performed isothermally at $325^{\circ} \mathrm{C}$. The friction coefficient between the cladding and the $\mathrm{Al}_{2} \mathrm{O}_{3}$ was assumed to be 0.55 as measured by Nakatsuka [21].

The calculated hoop stresses and strains on the inner surface of the cladding and over the $\mathrm{Al}_{2} \mathrm{O}_{3}$ interfaces are plotted in fig. 9 using the measured diametral strains as input to the calculations. Regular and thin-wall samples are modeled as plane stress tests and the chamfered sample is modeled as a plane strain test. The measured values of diametral strain at failure, i.e. $0.8 \%$ for the chamfered design and $2.0 \%$ for the regular design are used as failure criteria that have been observed experimentally. However, it is the calculated values of the local hoop stresses and strains that are of importance in the following analysis and discussion.

The local hoop strains for the regular specimen (modeled as plane stress) and the chamfered specimen (modeled as plane strain) are nearly identical at $0.8 \%$ measured diametral strain. Yet, at failure, the local hoop strain in the regular sample is over twice that in

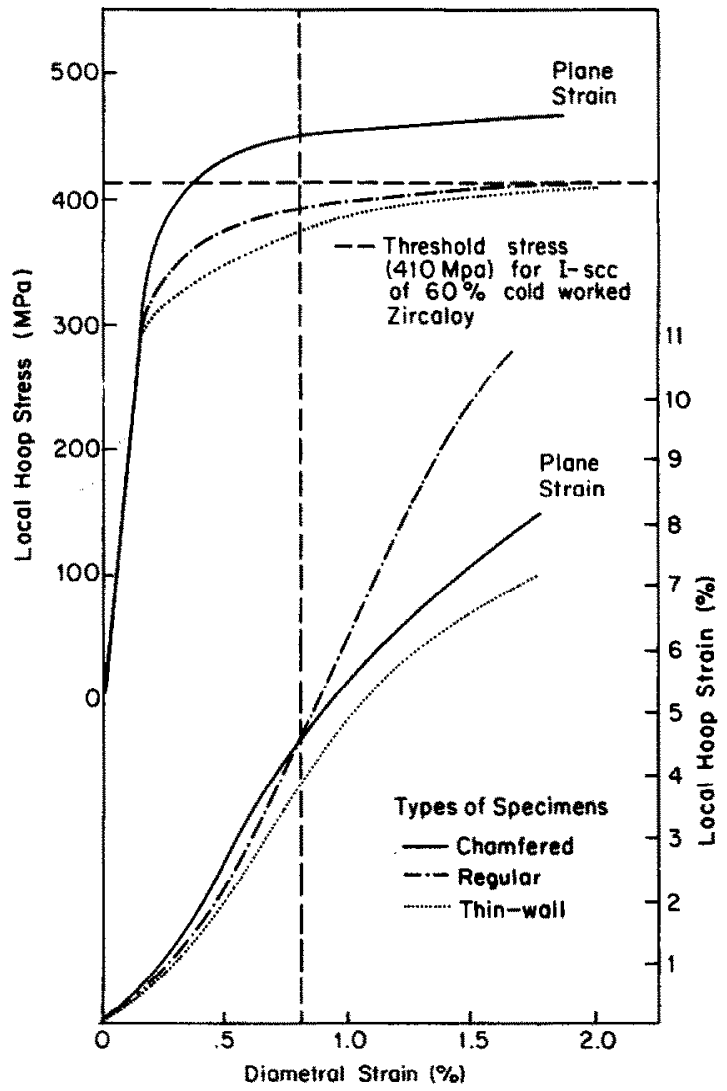

Fig. 9. Calculated local hoop stresses and strains on the inner surfaces of the three specimen designs at the segment interface as a function of the measured diametral strain at the midpoint of the $\mathrm{Al}_{2} \mathrm{O}_{3}$ segment. Results shown assume a plane stress state in the regular and thin-wall designs and a plane strain state in the chamfered design.

the chamfered sample. The calculated local hoop stress for the chamfered sample, however, is 10 to $15 \%$ greater than that for the regular and thin-wall samples in the range $0.8 \%$ and $2.0 \%$ diametral strain. These results have important consequences regarding the effect of the stress-strain state on failure strain and are discussed in the next section.

\section{Discussion}

The large difference in the calculated local strain at failure between the regular tubing sample and the chamfered design, as shown in fig. 9, suggests that the magnitude of the local hoop strain is not the determin- 
ing factor for failure by SCC. Inspection of the local hoop stress curves shows a strong correlation with failure propensity. If the chamfered samples are indeed subject to nearly plane strain whereas regular and thin-wall tubes are deformed in plane stress, then the significant difference in local hoop stress could explain the lower measured diametral failure strains.

Such differences are particularly relevant in view of some experimental results reported by Videm et al. [17]. By measuring the failure times of IPTT samples at different stresses, Videm and co-workers determined a SCC threshold stress of $410 \mathrm{MPa}$ for $60 \%$ cold-worked Zircaloys. As indicated in fig. 9, this threshold value (which is the minimum stress to cause failure in smooth specimens within a given time period) is exceeded by mandrel samples in a plane strain state at fairly low total measured diametral strains, but in the plane stress state it is not surpassed unless severe diametral straining occurs.

Videm et al. [17] also compared the I-SCC failure data for smooth IPTT specimens with those for tubes with fatigue-induced incipient cracks and concluded that the time, $T_{\mathrm{i}}(\mathrm{min})$, required to initiate a $70 \mu \mathrm{m}$ deep stress corrosion crack in $60 \%$ cold-worked material subjected to a hoop stress, $\sigma_{\theta}(\mathrm{MPa})$, is given by:

$T_{\mathrm{i}}=45 /\left(\sigma_{\theta}-410\right)^{0.6}, \quad \sigma_{\theta}>410 \mathrm{MPa}$.

Chamfered samples in plane strain states have calculated local hoop stresses greater than $410 \mathrm{MPa}$ for diametral strains larger than about $0.3 \%$, fig. 9. Between 0.3 and $0.8 \%$ (measured diametral strain) the average local hoop stress is approximately $430 \mathrm{MPa}$ which, from eq. (1), corresponds to $T_{\mathrm{i}}=75 \mathrm{~min}$. At an actuator speed of $12.7 \mu \mathrm{m} / \mathrm{min}$, it takes about $20 \mathrm{~min}$ to achieve $0.3 \%$ diametral strain, yielding a crack initiation time for chamfered specimens of $\sim 95 \mathrm{~min}$. Since the average failure time is $\sim 120 \mathrm{~min}$, it is not unreasonable that the crack nucleation period would constitute about $80 \%$ of the total failure time of chamfered tubes. This is consistent with the fracture morphology and related cracking rates discussed earlier.

An interesting comparison is presented in fig. 10 which shows SAF2D calculated hoop strain contours in actual fuel cladding and in a regular SEMT specimen using a finite-element model with 16 radial fuel cracks, a typical number in high burn-up $\mathrm{UO}_{2}$ pellets. The hoop strains in cladding subjected to an "instantaneous" power increase from $260 \mathrm{~W} / \mathrm{cm}$ to $460 \mathrm{~W} / \mathrm{cm}$ after 10 GWd/MTU burn-up are presented in fig. 10(a). Such severe ramping conditions have a high probability of inducing PCI failures in standard cladding in test reactor experiments [22]. Yet, the total diametral strain is

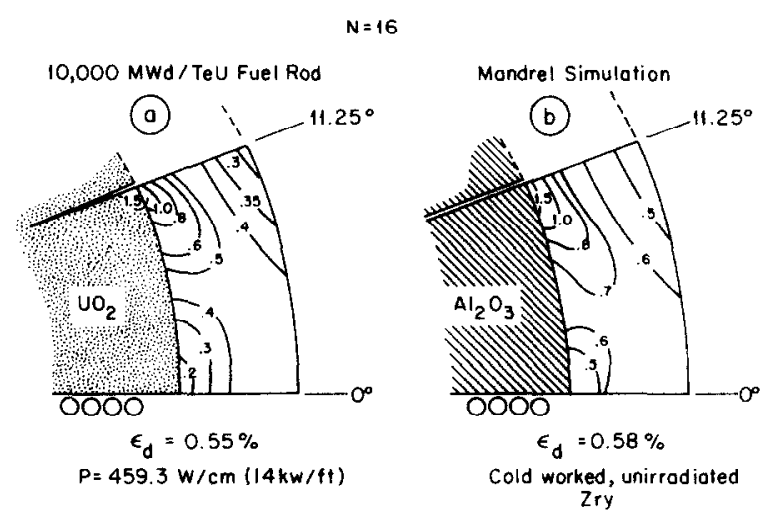

Fig. 10. Calculated hoop strain contours in (a) power-ramped fuel cladding, and (b) a regular SEMT sample. Sixteen radial cracks are modeled.

only $0.55 \%$ and the peak local hoop strain does not exceed $-1.5 \%$. These calculations show good agreement between the mandrel simulation and the PCI-defected rods.

The discrepancy between these results and those from SEMT tests is believed to be due mainly to the following differences: In mandrel simulations, four segments are displaced in four different directions whereas in fuel pellets a much higher number of fragments expand in all radial directions. The orthogunal movements of adjacent mandrel segments creates a type of "concentrated" loading that enhances bending moment effects and friction interactions near the segment interfaces. It has also been found that as the number of segments is increased from 4 to 16 the severity of the mandrel test with respect to the actual fuel behavior is practically eliminated. Therefore, the four-segmented mandrel test is actually expected to locally overstrain the tubing specimens when compared to fuel cladding deformed to similar average diametral strain. Whether such high strains are accompanied by correspondingly high stresses (which are sustained for sufficiently long times to cause failure) is dependent on the specimen stress-strain state as previously discussed.

The immediate implication is that local hoop strains imposed on SEMT specimens by four $\mathrm{Al}_{2} \mathrm{O}_{3}$ segments at relatively low diametral strains are in excess of what should be necessary to cause PCI cracking. However, both the local strain and the measured diametral strain at failure have been reduced significantly by imposing a semi-plane-strain condition. It is conceivable then, that if the test could be conducted in a more nearly pure plane strain condition, that these strains would drop substantially. 


\section{Conclusion}

It has been demonstrated experimentally that unirradiated, cold-worked chamfered Zircaloy- 2 specimens reproducibly fail at low $(0.8 \%)$ total diametral strains in ramp-hold tests in iodine at $325^{\circ} \mathrm{C}$. This strain level was arrived at by imposing a local stress state in the cladding that was initially designed to simulate the in-core situation. Regular and thin-wall specimens survive nominally identical test conditions. Regular specimens require considerably larger $(\geqslant 2.0 \%)$ diametral strains for failure than do chamfered specimens. Local strain measurements have indicated that the principal difference between these two specimen types is that the chamfered one is subjected to a near plane-strain condition whereas the uniform cladding is nearer to plane stress.

Finite-element analysis of these tests has shown that a substantial difference in local hoop stress exists between mandrel specimens loaded in plane-strain and plane-stress conditions. At $0.8 \%$ diametral strain the local hoop stress is on the average, $15 \%$ greater for the plane-strain state than for the plane-stress state, while the local hoop strains are nearly identical. On the basis of these results, it is suggested that failure is governed by the local hoop stress on the inner surface of the cladding wall.

\section{Acknowledgements}

The authors gratefully acknowledge R.P. Tucker, S.B. Wisner, G.H. Henderson, R.A. Rand and J.E. Lewis of the General Electric Company for their help in experiment design and data analysis.

\section{References}

[1] J.T.A. Roberts, Structural Materials in Nuclear Power Systems (Plenum Press, New York, 1981) pp. 53-85.
[2] F. Garzarolli, R. Manzel, M. Peeks and H. Stehle, Kerntechnik 20 (1978) p. 27.

[3] B.N. Nobrega, Ph.D. thesis, The University of Michigan, Ann Arbor (1984).

[4] E. Smith, in: Trans. 6th SMIRT Conference, Paris, 1981, (North-Holland, Amsterdam, 1981) paper D1/1.

[5] K. Videm and L. Lunde, Ann. Nucl. Energy 3 (1977) 305.

[6] H.M. Chung and F.L. Yaggee, in: 6th Int. Conf. on Zirconium in the Nuclear Industry, Vancouver, B.C. (July, 1983).

[7] L. Lunde and K. Videm, J. Nucl. Mater. 95 (1980) 210.

[8] R.L. Jones, D. Cubicciotti and B.C. Syrett, J, Nucl. Mater. $91(1980) 277$.

[9] B.C. Syrett, R.L. Jones and D. Cubicciotti, J. Nucl. Mater. 96 (1981) 160.

[10] H. Mogard, A. Aas and S. Junkrans, in: Proc. 4th IAEA Conf., Geneva, 10 (1971) 273.

[11] D.S. Tomalin, R.B. Adamson and R.P. Gangloff, in: Zirconium in the Nuclear Industry, ASTM STP 681 (American Society for Testing and Materials, 1979) p. 122.

[12] A. Garlick, J. Nucl. Mater. 49 (1973/74), 209.

[13] K. Une, J. Nucl. Sci.\&Technol. 14 (1977), 610.

[14] B.N. Nobrega, R.B. Adamson, J.S. King and G.S. Was, J. Nucl. Mater. 131 (1985) 99, this issue.

[15] L.F. Coffin and R.P. Gangloff, in: Water Reactor Fuel Performance, ANS Topical Meeting (St. Charles, IL, 1977) pp. 315-324.

[16] R.P. Gangloff, GEAP-2509, General Electric Company, (Nov. 1979).

[17] K. Videm, L. Lunde, T. Hollowell, K. Vilpponen and C. Vitanza, J. Nucl. Mater. 87 (1979) 259.

[18] R.L. Jones, R.L. Yaggee, R.A. Stoehr and D. Cubicciotti, J. Nucl. Mater. 82 (1979) 26.

[19] Y.R. Rashid, Proc. Conf. on Computational Methods in Nuclear Engineering (Charleston, SC, 1975).

[20] Y.R. Rashid, Nucl. Eng. and Design 29 (1974) 22.

[21] M. Nakatsuka, J. Nucl. Mater. 96 (1981) 205.

[22] H.S. Rosenbaum, GEAP-25163-6, General Electric Company (Feb. 1982). 